\title{
Graphene oxide: a stable carbon framework for functionalization $\uparrow$
}

Cite this: J. Mater. Chem. A, 2013, 1 11559

Received 30th July 2013

Accepted 15th August 2013

DOI: $10.1039 / c 3 t a 12975 f$

\author{
Siegfried Eigler, ${ }^{*}$ Stefan Grimm, Ferdinand Hof and Andreas Hirsch
}

www.rsc.org/MaterialsA

The effect of $\mathrm{NaOH}$ and $\mathrm{HCl}$ on the stability of the carbon framework in graphene oxide (GO) after substitution or etherification reaction in GO is demonstrated at $10^{\circ} \mathrm{C}$ and $40^{\circ} \mathrm{C}$, respectively. Our results allow the preparation of functionalized GO based architectures with an intact $\sigma$-framework of carbon atoms.

Graphene and its derivatives are some of the most versatile building blocks used for a variety of applications either in electronic devices or in medical applications. ${ }^{1-4}$ Graphene of the highest quality is preferably prepared by chemical vapour deposition or mechanical cleavage. ${ }^{5}$ However, these approaches suffer from high energy processes and the lack of chemical versatility. ${ }^{2}$ Thus, GO is used as a versatile precursor as it can be chemically functionalized easily. ${ }^{6} \mathrm{GO}$ is available from graphite and produced by wet chemical oxidation in sulphuric acid with potassium permanganate as an oxidant. ${ }^{7,8}$ The initially yielded graphite oxide can delaminate to single layers of GO. The main drawback of this conventionally prepared GO is the destructed structure of the carbon framework due to over oxidation..$^{\mathbf{9 1 0}}$ The carbon atoms in GO are functionalized with epoxy, hydroxyl and organosulfate as major groups. ${ }^{\mathbf{1 1}}$ The loss of carbon during synthesis and the accompanied rupture of the $\mathrm{sp}^{2}$-carbon lattice limit the overall quality of GO and the performance of graphene that is obtained after reduction. ${ }^{\mathbf{1 2 , 1 3}}$ Recently, we succeeded in making a new form of GO that can be reduced to graphene as a consequence of an intact carbon lattice. ${ }^{\mathbf{1 4}}$ After reduction the distance between defects was probed to be on the multi-nanometer scale and the performance in terms of charge carrier mobility was found to exceed $1000 \mathrm{~cm}^{2} \mathrm{~V}^{-1} \mathrm{~s}^{-1}$ for the best quality of flakes. Further on, this new form of GO can be used to visualize the efficiency of reducing agents. ${ }^{15}$

Department of Chemistry and Pharmacy and Institute of Advanced Materials and Processes (ZMP), Friedrich-Alexander-Universität Erlangen-Nürnberg (FAU), Dr.-Mack Str. 81, 90762 Fürth, Germany. E-mail: siegfried.eigler@fau.de; Fax: +49 (0)911 6507865015; Tel: +49 (0)9116507865005

$\uparrow$ Electronic supplementary information (ESI) available: Experimental procedures, UV-vis, FTIR spectra, and TG analysis. See DOI: 10.1039/c3ta12975f
One crucial question that could not be answered until now is about the stability of the carbon framework in GO during chemical transformation or simply is a dispersion of GO chemically stable to be used for applications. Dimiev et al. deeply describe how GO is degraded by water, especially by treatment with base. ${ }^{16}$ This decomposition finally leads to a material similar to humic acid as described by Staudenmaier before. ${ }^{17}$ Such a degradation in water would limit the usage of GO for the preparation of molecular architectures that need an undisturbed carbon framework. Until now, it has not been possible to demonstrate the stability of the carbon framework in GO after chemical reactions.

Here, we describe that the carbon framework of GO is maintained in water but degrades in the presence of base at elevated temperatures. For this purpose, graphene was obtained from $\mathrm{GO}$, either treated with $\mathrm{HCl}$ or treated with $\mathrm{NaOH}$ at $10{ }^{\circ} \mathrm{C}$ and $40{ }^{\circ} \mathrm{C}$, respectively. We probe the quality of $\mathrm{GO}$ after reduction (rGO) by statistical Raman spectroscopy. ${ }^{15,18}$ The carbon lattice is stable in GO treated by acid or base at $10{ }^{\circ} \mathrm{C}$. Despite that finding, it is ruptured by the treatment of base at $40{ }^{\circ} \mathrm{C}$. Furthermore, we find evidence for GO bearing predominately hydroxyl groups after base treatment at $10{ }^{\circ} \mathrm{C}$ and the formation of ether groups in water or acidic dispersion at $40{ }^{\circ} \mathrm{C}$ (Scheme 1). These insights make new molecular architectures feasible to extend the properties of graphene in the future.

It is well known that GO decomposes at elevated temperatures and GO was found to be metastable even at $25{ }^{\circ} \mathrm{C}$. $^{\mathbf{9 , 1 9 , 2 0}}$ Such instability hampers the functionalization of GO to date. To gain information about the chemical functionalization and the integrity of the carbon framework in GO we used our recently prepared new form of GO. ${ }^{14}$ Now, GO was mixed with $\mathrm{HCl}$ $(0.1 \mathrm{M})$ at $10{ }^{\circ} \mathrm{C}$ and $40{ }^{\circ} \mathrm{C}$, respectively. After that, the dispersion was purified by repeated centrifugation and redispersion in water until the $\mathrm{pH}$ of the supernatant was neutral $(\mathrm{GO}-\mathrm{HCl}$ and GO-HCl-40). The same procedure was used to prepare GO$\mathrm{NaOH}$ and GO-NaOH-40 using $\mathrm{NaOH}$ (0.1 M) instead of $\mathrm{HCl}$. While GO and GO-HCl appear to be yellowish, GO-NaOH turned brownish or grey (GO-NaOH-40) as a freeze-dried solid. 


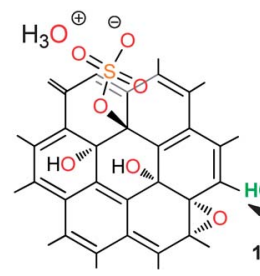
$\mathrm{GO}\left(10^{\circ} \mathrm{C}\right)$
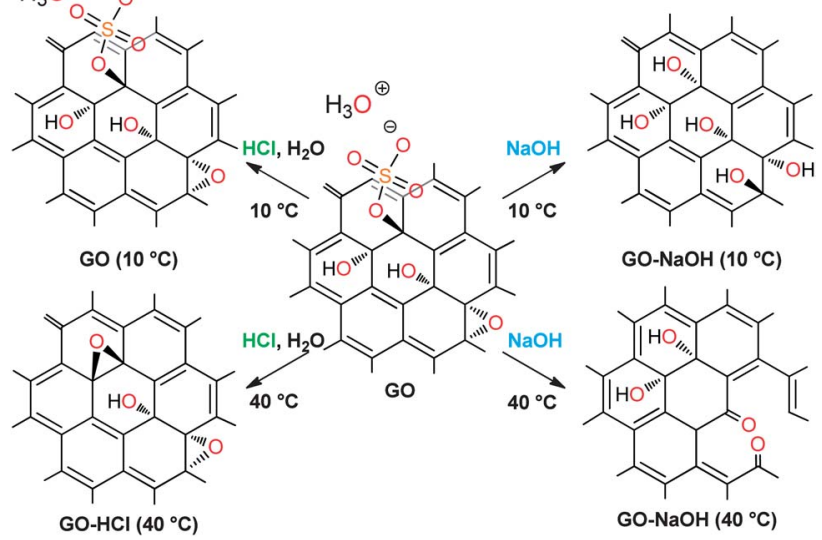

GO-NaOH $\left(10^{\circ} \mathrm{C}\right)$

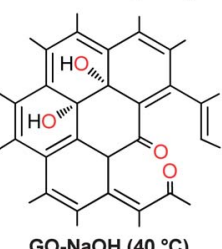

Scheme 1 Illustration of the reactivity of $\mathrm{GO}$ at $10{ }^{\circ} \mathrm{C}$ and $40{ }^{\circ} \mathrm{C}$, respectively, upon $\mathrm{HCl}$ or $\mathrm{NaOH}$ treatment; $\mathrm{GO}$ with hydroxyl, epoxy, $\mathrm{sp}^{2}$ regions and organosulfate (most likely with hydronium as counter-ion). ${ }^{11}$

These results are also reflected by the respective UV-vis spectra that are almost identical for GO, GO-40, GO-HCl and GO-HCl40. GO-NaOH shows an increased absorption between 400 and $600 \mathrm{~nm}$, which is an indication for an increased $\pi$-system as a consequence of the cleavage of organosulfate or elimination of functional groups (Fig. $\mathrm{S} 1 \dagger$ ). These spectral changes are much more pronounced for $\mathrm{GO}-\mathrm{NaOH}-40$ as a consequence of an enlarged heterogeneous structure. ${ }^{\mathbf{1 1}}$ Fourier transform infrared (FTIR) spectroscopy on ZnSe windows was used to elucidate changes in the chemical structure.

For GO and GO-HCl (treated at $10{ }^{\circ} \mathrm{C}$ ), there is almost no change in FTIR spectra visible indicating the intact chemical structure (Fig. S2 $\dagger$ ). Thus, we conclude that there is no reaction occurring. After $\mathrm{NaOH}$ treatment at $10{ }^{\circ} \mathrm{C}$ there are significant changes $\left(1626,1384\right.$, and $\left.1249 \mathrm{~cm}^{-1}\right)$ that come along with the cleavage of organosulfate as found by TG-MS analysis (Fig. 1, $\mathrm{S} 3 \dagger) .{ }^{11}$ Treatment of GO at $40{ }^{\circ} \mathrm{C}$ in water results in a dramatic change of FTIR spectra. Thus, for GO-40 we find only small signals at about $1250 \mathrm{~cm}^{-1}$ that had been dominant in $\mathrm{GO}$ and an increased signal intensity at $1074 \mathrm{~cm}^{-1}$ (Fig. S2 $\dagger$ ). At 1074 $\mathrm{cm}^{-1}$, vibrations generally originate from $\mathrm{C}-\mathrm{O}$ or $\mathrm{C}-\mathrm{C}$ skeleton vibrations. ${ }^{19}$ In particular, for GO-HCl-40 this signal dominates the spectrum (Fig. 1) and thus, a structure with an enhanced amount of ether groups is formed, as epoxy groups or other ethers as proposed in the Ruess model. ${ }^{21}$ However, to date the structural analysis of differently treated GO is limited and several structure models have been proposed and proved in the literature. ${ }^{16,22,23}$ However, more analysis appears to be necessary in future studies. For GO-NaOH-40 the FTIR spectra indicate the degradation of the material as described in the literature. ${ }^{\mathbf{1 6}}$ Generally, broad signals are found that stem from a less defined structure also indicated by Raman spectroscopy (Fig. S2†).

We further analyzed the materials by thermogravimetric (TG) analysis. For GO-NaOH, GO-40, GO-HCl-40 and GO-NaOH-40 organosulfate is cleaved as indicated by the missing weight-loss step between 200 and $300{ }^{\circ} \mathrm{C}$ that relates to decomposing organosulfate. ${ }^{11}$ Thus, we conclude that organosulfate remains stable at $10{ }^{\circ} \mathrm{C}$ in the absence of base. Further evidence for the

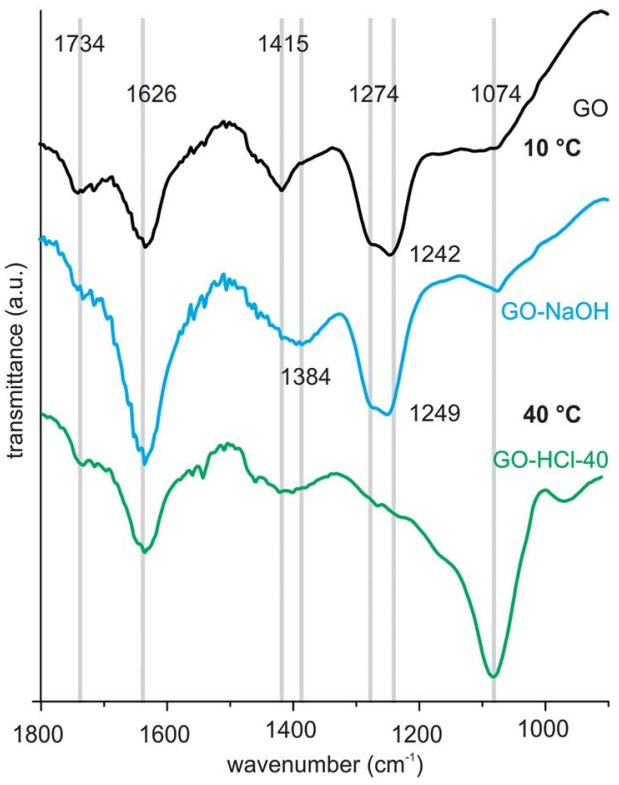

Fig. 1 FTIR spectra of $\mathrm{GO}, \mathrm{GO}-\mathrm{NaOH}$ and $\mathrm{GO}-\mathrm{HCl}-40$ in the region between $1800 \mathrm{~cm}^{-1}$ and $900 \mathrm{~cm}^{-1}$ (see ESI†).

Table 1 Quality of treated GO determined by Raman spectra analysis

\begin{tabular}{lrrll}
\hline Material & $I_{\mathrm{D}} / I_{\mathrm{G}}$ & $\Gamma_{2 \mathrm{D}}\left(\mathrm{cm}^{-1}\right)$ & C-frame $^{a}$ & Modified $^{b}$ \\
\hline GO & $2.7 \pm 0.3$ & $69 \pm 13$ & Stable & No \\
GO-HCl & $2.6 \pm 0.3$ & $66 \pm 12$ & Stable & No \\
GO-NaOH & $2.5 \pm 0.3$ & $73 \pm 17$ & Stable & Yes \\
GO-40 & $2.7 \pm 0.3$ & $72 \pm 12$ & Stable & Yes \\
GO-HCl-40 & $2.7 \pm 0.3$ & $72 \pm 12$ & Stable & Yes \\
GO-NaOH-40 & $1.9 \pm 0.2$ & $120 \pm 25$ & Not stable & Yes
\end{tabular}

${ }^{a}$ Stability of the carbon framework according to Raman spectroscopy. ${ }^{b}$ Chemically modified according to TG analysis or FTIR analysis.

stability of organosulphate at low $\mathrm{pH}$ and its cleavage at high $\mathrm{pH}$ or $40{ }^{\circ} \mathrm{C}$ is found by elemental analysis, especially according to the sulphur content (Table $\mathrm{S} 1 \dagger$ ). These analyses reveal that $\mathrm{NaOH}$ treatment of GO causes likely a nucleophilic reaction of hydroxide at $\mathrm{sp}^{3}$-carbon centers with sulfate as the leaving group (Scheme 1). Hydroxy-GO can efficiently be reduced to rGO, because hydroxyl groups are natively present in GO. Besides, other reactions, as ring opening of epoxy groups or irreversible base induced rearrangement reactions, are also feasible as proposed earlier. ${ }^{19}$

Statistical Raman spectroscopy was used to gain information about the integrity of the carbon framework. To evaluate the defect density by scanning Raman spectroscopy we prepared thin films on $\mathrm{Si} / \mathrm{SiO}_{2}$ wafers by the Langmuir-Blodgett technique as described earlier and reduced them to rGO by vapor treatment with hydriodic acid and trifluoro acetic acid. ${ }^{\mathbf{1 4 2 4}}$ Firstly, AFM images of the graphene films were prepared (Fig. S4 $\dagger$ ) and differences in analyzed flake thickness between 1.5 and $0.7 \mathrm{~nm}$ were observed with the lowest thickness for rGO-NaOH. We suggest that base treatment of GO and the accompanied cleavage of organosulfate are responsible for the reduced thickness. 

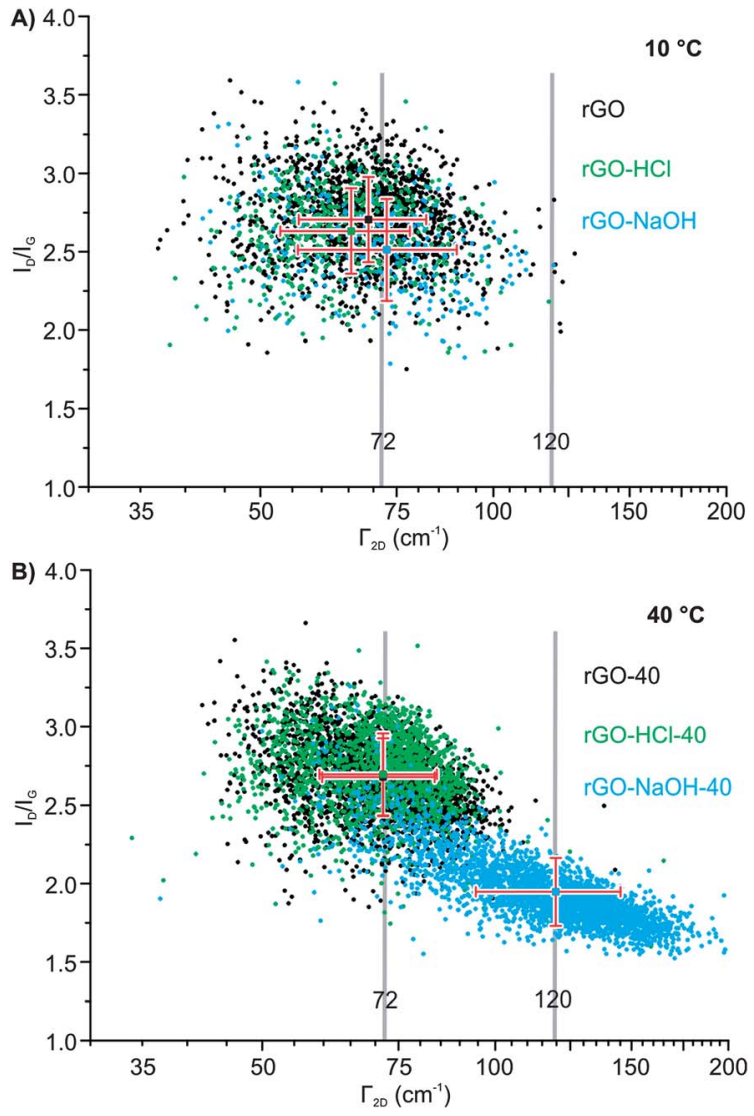

Fig. 2 Raman analysis of the overall quality of (A) $r G O, r G O-H C l$, and $r G O-N a O H$ and (B) rGO-40, rGO-HCl-40 and rGO-NaOH-40.

Next, an area of $22500 \mu \mathrm{m}^{2}$ of these films was probed by Raman spectroscopy with an increment of $2.5 \mu \mathrm{m}$. To ensure that Raman spectra of single layers are predominantly analyzed, we filtered the spectra by the intensity of the G peak to exclude spectra from the multilayered material. With this technique in hand defect densities can be easily probed. ${ }^{15,18}$ Therefore, the intensity and the full width at half maximum $(T)$ of the defect induced $\mathrm{D}$ peak, the G peak and the $2 \mathrm{D}$ peak were analyzed. As shown before, the plot of $I_{\mathrm{D}} / I_{\mathrm{G}}$ against $\Gamma_{2 \mathrm{D}}$ makes the quality of rGO visible. ${ }^{14}$ The $\Gamma_{2 \mathrm{D}}$ depends strongly on the preparation conditions of GO and the reduction conditions. ${ }^{15}$ Thus, $\Gamma_{2 \mathrm{D}}$ varies between $30 \mathrm{~cm}^{-1}$ for pristine graphene and up to 250 $\mathrm{cm}^{-1}$ for thermally reduced GO. ${ }^{15}$ In this study we determined the quality of rGO reflected by the $I_{\mathrm{D}} / I_{\mathrm{G}}$ ratio of $2.7 \pm 0.3$ and the $\Gamma_{2 \mathrm{D}}$ of $66 \pm 15 \mathrm{~cm}^{-1}$, on average (Table 1 and Fig. 2). For rGO$\mathrm{HCl}$ and $\mathrm{rGO}-\mathrm{NaOH}$ we find almost the same quality with $\Gamma_{2 \mathrm{D}} \approx 70 \mathrm{~cm}^{-1}$. These data reveal that the carbon framework was not further harmed by $\mathrm{HCl}$ and $\mathrm{NaOH}$ treatment at $10{ }^{\circ} \mathrm{C}$, respectively. Surprisingly, the quality of rGO-40 is the same as for rGO, even if treated at $40{ }^{\circ} \mathrm{C}$ (Table 1), despite a modified structure as indicated by FTIR. In contrast to that, the collapse of the carbon $\mathrm{sp}^{2}$-framework is evident from the analysis of rGO-NaOH-40 with a broad $\Gamma_{2 \mathrm{D}}$ of $120 \pm 25 \mathrm{~cm}^{-1}$.

Therefore, as already indicated by UV-vis and FTIR spectra hydroxide treatment of $\mathrm{GO}$ at $40{ }^{\circ} \mathrm{C}$ results in the degradation of the carbon framework yielding graphene with a more amorphous structure. This observation is likely due to $\mathrm{CO}_{2}$ formation or rearrangement reactions starting at highly oxidized regions as proposed by Dimiev et al. ${ }^{16,19}$ Surprisingly, the treatment of $\mathrm{GO}$ with $\mathrm{NaOH}$ at $10{ }^{\circ} \mathrm{C}$ does not harm the carbon framework significantly, even if organosulfate is cleaved (Scheme 1, Fig. 2, Table 1). Thus, we conclude that the defect density in rGO remains constant for samples treated with acid or base at $10^{\circ} \mathrm{C}$ or treated at $40{ }^{\circ} \mathrm{C}$ in pure water or $\mathrm{HCl}$. Earlier studies about the stability of GO in acid were conducted at room temperature and they indicate a slow degradation of GO. ${ }^{16}$

We conclude that the $\sigma$-framework of carbon atoms in GO is stable in water and $\mathrm{HCl}$ at $10{ }^{\circ} \mathrm{C}$ and $40{ }^{\circ} \mathrm{C}$, respectively. In addition, $\mathrm{GO}$ is stable upon $\mathrm{NaOH}$ treatment at $10^{\circ} \mathrm{C}$, but not at $40^{\circ} \mathrm{C}$. We find evidence for the change of the chemical structure of GO if treated with base at $10{ }^{\circ} \mathrm{C}$ whereby organosulfate is likely substituted by hydroxide. Moreover, at $40{ }^{\circ} \mathrm{C}$ the cleavage of organosulfate is indicated in water and under acidic conditions. For $\mathrm{GO}-\mathrm{HCl}-40$ etherification is implied by $\mathrm{C}-\mathrm{O}$ vibrations at $1074 \mathrm{~cm}^{-1}$. Despite these chemical modifications of the structure of GO, it can be reduced to the same quality of graphene as proved by Raman spectroscopy which indicates the preserved carbon framework. In contrast, for GO treated with $\mathrm{NaOH}$ at $40{ }^{\circ} \mathrm{C}$, we find a structure that is dominated by defects after reduction, due to the rupture of the carbon framework. This degradation of GO leads to a material that is alike humic acids as already realized by Staudenmaier et al. among others., ${ }^{95-17,19}$ Any instability of GO would exclude its functionalization for the preparation of reduced functionalized GO with superior electronic properties in the future. Thus, we proved the ability to functionalize GO preserving the $\sigma$-framework of carbon atoms. More knowledge about the chemical structure of GO is necessary to clarify the structural changes possible in GO. Furthermore, long term investigations on the stability of GO are necessary since commercial products need to be stable at least on the month scale. Moreover, reactions must be identified that proceed at low temperature to safely prepare functionalized GO and functionalized graphene, respectively.

\section{Acknowledgements}

The authors thank the Deutsche Forschungsgemeinschaft (DFG - SFB 953, Project A1 "Synthetic Carbon Allotropes"), the European Research Council (ERC; grant 246622 - GRAPHENOCHEM), and the Cluster of Excellence 'Engineering of Advanced Materials (EAM)' for financial support.

\section{Notes and references}

1 V. Georgakilas, M. Otyepka, A. B. Bourlinos, V. Chandra, N. Kim, K. C. Kemp, P. Hobza, R. Zboril and K. S. Kim, Chem. Rev., 2012, 112, 6156-6214.

2 K. S. Novoselov, V. I. Fal'ko, L. Colombo, P. R. Gellert, M. G. Schwab and K. Kim, Nature, 2012, 490, 192-200.

3 C. Chung, Y. K. Kim, D. Shin, S. R. Ryoo, B. H. Hong and D. H. Min, Acc. Chem. Res., 2013, DOI: 10.1021/ar300159f. 
4 H. Y. Mao, S. Laurent, W. Chen, O. Akhavan, M. Imani, A. A. Ashkarran and M. Mahmoudi, Chem. Rev., 2013, 113, 3407-3424.

5 C. Mattevi, H. Kim and M. Chhowalla, J. Mater. Chem., 2011, 21, 3324-3334.

6 D. Chen, H. Feng and J. Li, Chem. Rev., 2012, 112, 6027-6053. 7 G. Charpy, C. R. Hebd. Seances Acad. Sci., 1909, 148, 920-923.

8 J. William, S. Hummers and R. E. Offeman, J. Am. Chem. Soc., 1958, 80, 1339.

9 S. Eigler, C. Dotzer and A. Hirsch, Carbon, 2012, 50, 3666-3673.

10 A. Dimiev, D. V. Kosynkin, L. B. Alemany, P. Chaguine and J. M. Tour, J. Am. Chem. Soc., 2012, 134, 2815-2822.

11 S. Eigler, C. Dotzer, F. Hof, W. Bauer and A. Hirsch, Chem.Eur. J., 2013, 19, 9490-9496.

12 S. Pei and H.-M. Cheng, Carbon, 2012, 50, 3210-3228.

13 S. Mao, H. Pu and J. Chen, RSC Adv., 2012, 2, 2643-2662.

14 S. Eigler, M. Enzelberger-Heim, S. Grimm, P. Hofmann, W. Kroener, A. Geworski, C. Dotzer, M. Rockert, J. Xiao, C. Papp, O. Lytken, H. P. Steinruck, P. Muller and A. Hirsch, Adv. Mater., 2013, 25, 3583-3587.
15 S. Eigler, Chem. Commun., 2013, 49, 7391-7393.

16 A. M. Dimiev, L. B. Alemany and J. M. Tour, ACS Nano, 2013, 7, 576-588.

17 L. Staudenmaier, Ber. Dtsch. Chem. Ges., 1899, 32, 28242834.

18 J. M. Englert, P. Vecera, K. C. Knirsch, R. A. Schafer, F. Hauke and A. Hirsch, ACS Nano, 2013, 7, 5472-5482.

19 S. Eigler, C. Dotzer, A. Hirsch, M. Enzelberger and P. Müller, Chem. Mater., 2012, 24, 1276-1282.

20 S. Kim, S. Zhou, Y. Hu, M. Acik, Y. J. Chabal, C. Berger, W. de Heer, A. Bongiorno and E. Riedo, Nat. Mater., 2012, 11, 544-549.

21 G. Ruess, Monatsh. Chem., 1947, 76, 381-417.

22 A. Lerf, H. He, M. Forster and J. Klinowski, J. Phys. Chem. B, 1998, 102, 4477-4482.

23 W. Cai, R. D. Piner, F. J. Stadermann, S. Park, M. A. Shaibat, Y. Ishii, D. Yang, A. Velamakanni, S. J. An, M. Stoller, J. An, D. Chen and R. S. Ruoff, Science, 2008, 321, 1815-1816.

24 P. Cui, J. Lee, E. Hwang and H. Lee, Chem. Commun., 2011, 47, 12370-12372. 